\title{
A MULTIPHASE, RIEMANN-SOLVER APPROACH TO GAS-CAVITATION
}

\author{
Andris Rambaks*, Hubertus Murrenhoff, Katharina Schmitz \\ Institute for Fluid Power Drives and Systems, Campus-Boulevard 30, Aachen 52074, Germany
}

\begin{abstract}
In hydraulic systems, a certain amount of air is always present as entrained air in the form of gaseous bubbles and as dissolved air. Large amounts of entrained air negatively affect the behavior of hydraulic systems by decreasing the bulk modulus of the fluid and increasing the risk of cavitation damage, e.g., cavitation erosion and the microdiesel effect. The diffusion-driven growth of entrained gaseous bubbles surrounded by a liquid phase is referred to as gas-cavitation, in which dissolved air from the solution diffuses into the bubble. In the proposed paper, the diffusion-driven growth of a single gas bubble immersed in a liquid is examined. To that end, a comprehensive literature review, a mathematical description of the problem as well as the numerical implementation is presented.
\end{abstract}

KEY WORDS: Bubble dynamics, Cavitation, Riemann-Solver, Fractional-Step-Method, Ghost-Fluid-Method, Level-Set-Method

\section{INTRODUCTION}

It is known that a liquid (solvent) can solve a certain amount of gas (solute) and form a homogeneous mixture (solution) [1]. In many cases, the liquid mixture behaves like an ideal dilute solution and can thus be described by Henry's law, according to which the fraction of the amount of substance of solute in the solution $\bar{x}_{i}^{l}$ is directly proportional to the partial pressure of the solute in the gaseous phase [1]:

$$
\bar{x}_{i}^{g} \cdot p^{g}=H_{i, l} \cdot \bar{x}_{i}^{l}
$$

Under certain circumstances, dissolved gas can become undissolved and form gas bubbles inside the liquid phase. These bubbles can grow and subsequently collapse in a process known as cavitation [2]. Based on the physical principle behind bubble growth, three distinct models for cavitation have been defined.

The first and simplest cavitation model is that of pseudo-cavitation, which is based on the Rayleigh-PlessetEquation (2). In this model, the bubble is assumed spherical, the pressure and temperature distribution inside the bubble is assumed uniform [2]. According to equation (2), changes in the external pressure $p_{\infty}(t)$ are the driving-force behind bubble growth (or shrinkage).

$$
\frac{p_{B}(t)-p_{\infty}(t)}{\rho_{L}}=R \frac{d^{2} R}{d t^{2}}+\frac{3}{2}\left(\frac{d R}{d t}\right)^{2}+\frac{4 v_{L}}{R} \frac{d R}{d t}+\frac{2 \sigma_{t}}{\rho_{L} R}
$$

The second cavitation model is that of vapor-cavitation which occurs, when the static pressure of the liquid drops below its saturation pressure and vapor bubbles are formed locally. This is a phenomenon most notably observed at high rotational speeds of ship's propellers [2]. 
The third cavitation model is that of diffusion-driven growth of entrained gaseous bubbles surrounded by a liquid phase, in which dissolved air from the solution diffuses into the bubbles. According to Brennen [2], if changes in the flow variables, e.g. pressure and temperature, do not occur rapidly, then the effects of mass diffusion must be accounted for.

In hydraulic systems, the contaminating gas is air, i.e. a mixture mainly made up of $\mathrm{O}_{2}$ and $\mathrm{N}_{2}$. Dissolved air generally has no effect on the properties of the hydraulic fluid, whereas large amounts of entrained air negatively affect the behavior of hydraulic systems by decreasing the bulk modulus of the fluid and increasing the risk of cavitation damage, e.g., cavitation erosion (Fig. 1) and the micro-diesel effect [3].

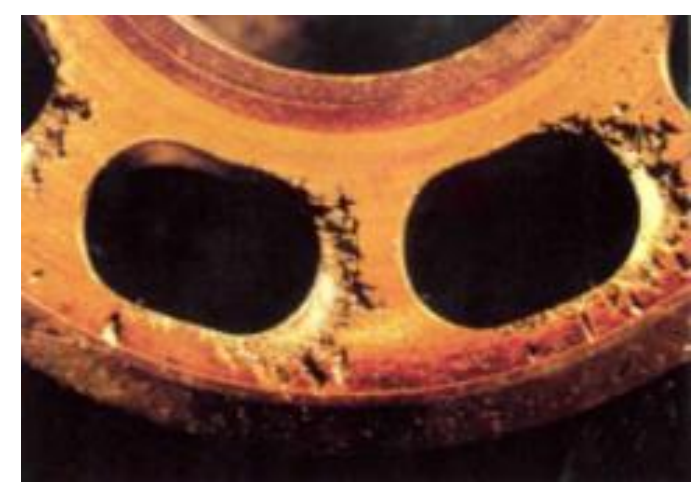

Fig. 1 Cavitation erosion in a hydraulic pump

Although the phenomenon of cavitation is occurring frequently, only pseudo- and vapor-cavitation are well understood. On the other hand, gas-cavitation is not fully understood. The aim of this paper is to present a numerical simulation model on the diffusion-driven growth of gas bubbles suspended in a liquid phase. First, the Navier-Stokes equations are derived for both the liquid and the gaseous phase and coupled at the interface. Secondly, the numerical scheme and its building blocks are presented.

\section{MATHEMATICAL DESCRIPTION}

To study the phenomenon of gas-cavitation, a physical system must be defined, and its mathematical description derived. As the emphasis is placed on studying the diffusion-driven growth of gas bubbles, it is of advantage to observe only a single gas bubble. Thus, coalescence effects are neglected. The resulting twophase system is depicted in Fig. 2., where $\boldsymbol{U}_{1}$ is the conserved variable vector of the gaseous phase and $\boldsymbol{U}_{2}$ of the liquid phase.

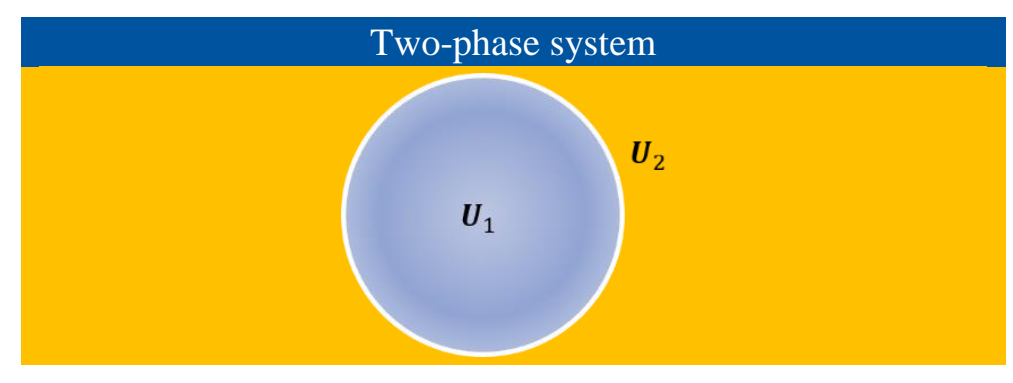

Fig. 2 Gas bubble surrounded by a liquid phase

To model gas bubble growth, it is justified to use an axisymmetric coordinate system, if the flow field around the bubble is at most transversal and if no flow separation occurs. This allows to use a two-dimensional coordinate system, thereby reducing the complexity of the model and computational time significantly. 


\subsection{Navier-Stokes equations}

Both gaseous and liquid phase are described by the two-dimensional, compressible, multicomponent NavierStokes equations given by equation (3). On the left side the first term, $\boldsymbol{U}$, is the conserved variable vector, $\boldsymbol{F}$ is the hyperbolic flux vector in X-direction and $\boldsymbol{G}$ is the hyperbolic flux vector in y-direction. On the right side the first term, $\boldsymbol{F}_{\text {par }}$, is the parabolic flux vector in x-direction, $\boldsymbol{G}_{\text {par }}$ is the parabolic flux vector in y-direction, $\boldsymbol{S}_{\text {geom }}$ is the geometric source term vector and $\boldsymbol{S}_{\text {chem }}$ is the chemical reaction source term vector.

$$
\frac{\partial \boldsymbol{U}}{\partial t}+\frac{\partial \boldsymbol{F}}{\partial x}+\frac{\partial \boldsymbol{G}}{\partial y}=\frac{\partial \boldsymbol{F}_{\text {par }}}{\partial x}+\frac{1}{y^{\zeta}} \frac{\partial y^{\zeta} \boldsymbol{G}_{\text {par }}}{\partial y}-\zeta \frac{\boldsymbol{S}_{\text {geom }}}{y}+\boldsymbol{S}_{\text {chem }}
$$

The components of the vectors are summarized in equation (4). The conserved variable vector $\boldsymbol{U}$ and the hyperbolic flux vectors $\boldsymbol{F}$ and $\boldsymbol{G}$ represent pure advection, whereas the parabolic flux vectors $\boldsymbol{F}_{\text {par }}, \boldsymbol{G}_{\text {par }}$ and the chemical reaction source term vector $\boldsymbol{S}_{\text {chem }}$ account for such phenomena as diffusion, viscosity, heat transfer and species production. The geometric source term vector $\boldsymbol{S}_{\text {geom }}$ always arises if a Cartesian coordinate system is not used.

$$
\begin{gathered}
\boldsymbol{U}=\left[\begin{array}{c}
x_{1} \rho \\
\vdots \\
x_{n} \rho \\
\rho u \\
\rho v \\
E
\end{array}\right], \boldsymbol{F}=\left[\begin{array}{c}
x_{1} u \rho \\
\vdots \\
x_{n} u \rho \\
\rho u^{2}+p \\
\rho u v \\
u(E+p)
\end{array}\right], \boldsymbol{G}=\left[\begin{array}{c}
x_{1} v \rho \\
\vdots \\
x_{n} v \rho \\
\rho u v+p \\
\rho v^{2}+p \\
v(E+p)
\end{array}\right], \boldsymbol{S}_{g e o m}=\left[\begin{array}{c}
x_{1} v \rho \\
\vdots \\
x_{n} v \rho \\
\rho v u \\
\rho v^{2} \\
v(E+p)
\end{array}\right], \boldsymbol{G}_{\text {par }}=\left[\begin{array}{c}
-j_{y, 1} \\
\vdots \\
-j_{y, n} \\
\sigma_{y x} \\
\sigma_{y y} \\
\vdots \\
-j_{x, n} \\
\sigma_{x x} \\
\sigma_{x y} \\
u \sigma_{x x}+v \sigma_{x y}-\dot{q}_{x}
\end{array}\right], \boldsymbol{S}_{\text {chem }}=\left[\begin{array}{c}
\dot{\omega}_{1} \\
\vdots \\
\dot{\omega}_{n} \\
0 \\
0 \\
0
\end{array}\right]
\end{gathered}
$$

To switch between Cartesian and axisymmetric coordinates, a coordinate selector variable $\zeta$ is defined by:

$$
\zeta= \begin{cases}0 & \text { for Cartesian coordinates } \\ 1 & \text { for axissymetric coordinates }\end{cases}
$$

To solve equation (3) another equation is needed as the system of equations is underdetermined. Because the flow studied is assumed compressible, thermodynamic relations are used. For the gaseous phase, the ideal gas law is used to derive an expression for the total energy $E_{g}$ but other relations like the covolume or van der Vaals can also be used [4]. The expression for the total energy of an ideal gas $E_{g}$ is given by:

$$
E_{g}=\frac{\rho}{2} \cdot u^{2}+\frac{p}{\gamma_{g}-1}
$$

For the liquid phase, the Tait equation of state is used to derive an expression for the total energy $E_{l}$ given by:

$$
E_{l}=\frac{\rho}{2} \cdot u^{2}+\frac{B \cdot \rho^{\gamma_{l}}}{\left(\gamma_{l}-1\right) \cdot \rho_{0}}+(B-A)
$$

The Navier-Stokes equations given by equation (3) are valid only in each respective phase. This becomes apparent when both terms for the total energy are examined. To couple the two phases, appropriate jump conditions at the interface must be defined. 


\subsection{Jump conditions at a moving interface}

At an interface between two phases, discontinuities can arise, e.g. due to surface tension and phase change [5]. When studying multiphase flow, these phenomena have to be accounted for. To that end, a control volume is defined across the interface as depicted in Fig. 3.

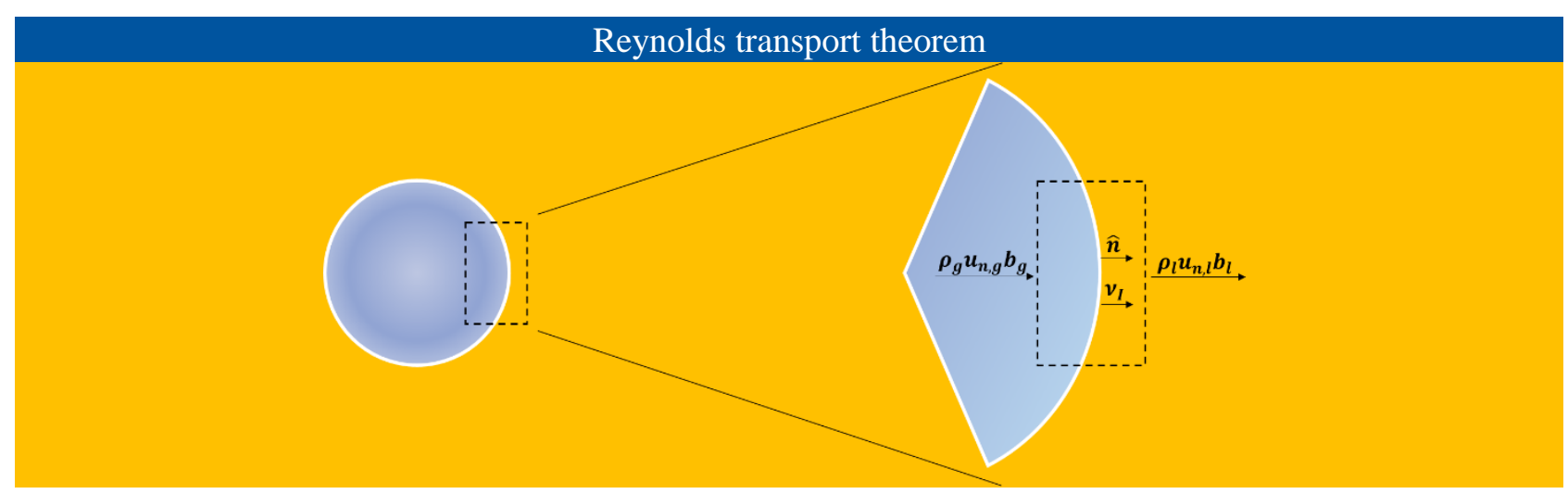

Fig. 3 Reynolds transport theorem at an interface

According to Mamoru et al. [6], at an interface between two phases, discontinuities arise and appropriate jump conditions have to specified, which relate the governing field equations to each other. Mathematically, a jump condition is defined as:

$$
[\Psi]:=\Psi_{g}-\Psi_{l}
$$

To determine the appropriate jump conditions at an interface, it is useful to make use of Reynolds transport theorem. It establishes a mathematical description between a system and a control volume and is given by equation (9), where $B_{s y s}$ is an extensive property of the system and $b$ is an intensive property of the system.

$$
\frac{D B_{s y s}}{D t}=\oiiint_{C V} \frac{\partial \rho b}{\partial t} d V+\oiint_{C S} \rho b\left(\boldsymbol{v}-\boldsymbol{v}_{I}\right) \cdot \widehat{\boldsymbol{n}} d A
$$

At an interface, there can be no agglomeration of any extensive property $B_{\text {sys }}$ because it is treated as a singular surface and the size of the control volume tends to zero [6]. Also, any fluxes entering the control volume perpendicular to the normal unit vector can be neglected [6]. Therefor equation (9) simplifies to:

$$
\frac{D B_{\text {sys }}}{D t}=\left[-\rho_{g} b_{g}\left(u_{n, g}-u_{n, I}\right)+\rho_{l} b_{l}\left(u_{n, l}-u_{n, I}\right)\right] \cdot A
$$

The velocity in normal direction is given by:

$$
u_{n}:=\boldsymbol{v} \cdot \widehat{\boldsymbol{n}}
$$

To derive appropriate jump conditions at an interface, an interfacial mass balance, an interfacial normal and tangential momentum balance, an interfacial mass species balance and an interfacial energy balance are made. Also, an in interfacial normal and tangential force balance is made. 
Interfacial mass balance. The mass balance at an interface is depicted in Fig. 4.

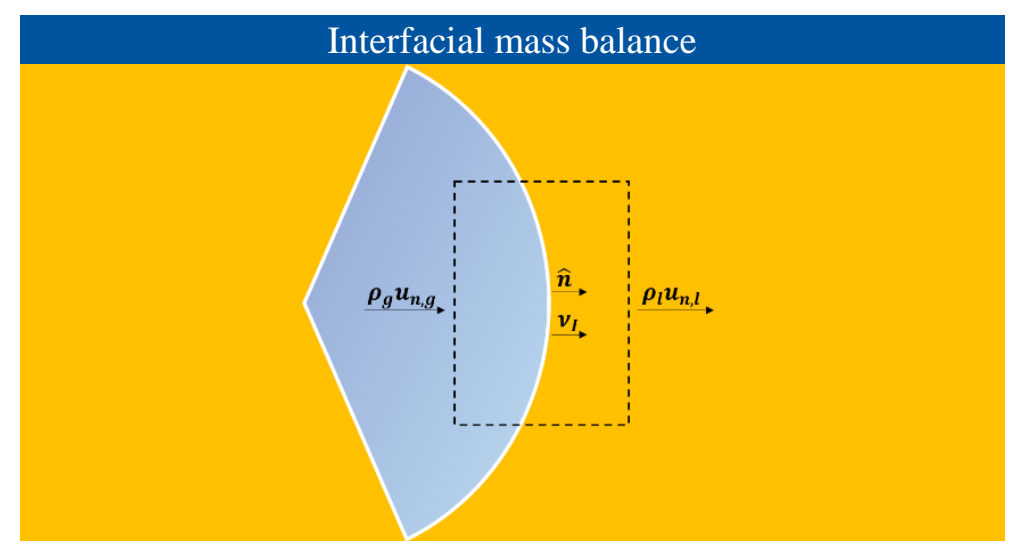

Fig. 4 Interfacial mass balance

Using equation (10) and setting $b=1$ results in the equation of continuity at an interface and is given by:

$$
-\rho_{g}\left(u_{n, g}-u_{n, I}\right)+\rho_{l}\left(u_{n, l}-u_{n, I}\right)=0
$$

In the case of condensation or evaporation at the interface, a regression rate $r_{b}$ can be defined as:

$$
r_{b}:=u_{n, l}-u_{n, I}
$$

Using equation (12) and (13) and by defining the interface mass flux with $\dot{m}_{I}^{\prime \prime}$, the expression for the regression rate can be rewritten to:

$$
r_{b}=\frac{\rho_{g}\left(u_{n, g}-u_{n, I}\right)}{\rho_{l}}=\frac{\dot{m}_{I}^{\prime \prime}}{\rho_{l}}
$$

Using equation (8), (13) and (14) an expression for the jump in normal velocity can be derived:

$$
\left[u_{n}\right]:=u_{n, g}-u_{n, l}=\left[\frac{1}{\rho}\right] \dot{m}_{I}^{\prime \prime}
$$

Interfacial normal momentum balance. The normal momentum balance and the normal force balance at an interface is depicted in Fig. 5. Using Reynolds transport theorem and Newton's second law, which states that a change in momentum is equal to the sum of all forces acting on the system, equation (16) is derived:

$$
-\rho_{g} u_{n, g}\left(u_{n, g}-u_{n, I}\right)+\rho_{l} u_{n, l}\left(u_{n, l}-u_{n, I}\right)=\left(p_{g}-\sigma_{n n, g}\right)-\left(p_{l}-\sigma_{n n, l}\right)-\kappa_{c} \sigma_{t}
$$

Using the definition in the jump in normal velocity and the definition of the regression rate, the jump in pressure across an interface is found:

$$
[p]=\kappa_{c} \sigma_{t}-\rho_{l} r_{b}\left[u_{n}\right]+\left[\sigma_{n n}\right]
$$

The first term on the right side of equation (17) represents the pressure difference due to surface tension, the second term is the recoil pressure due to phase change at the interface and the third term is the jump in normal deviatoric stress. 


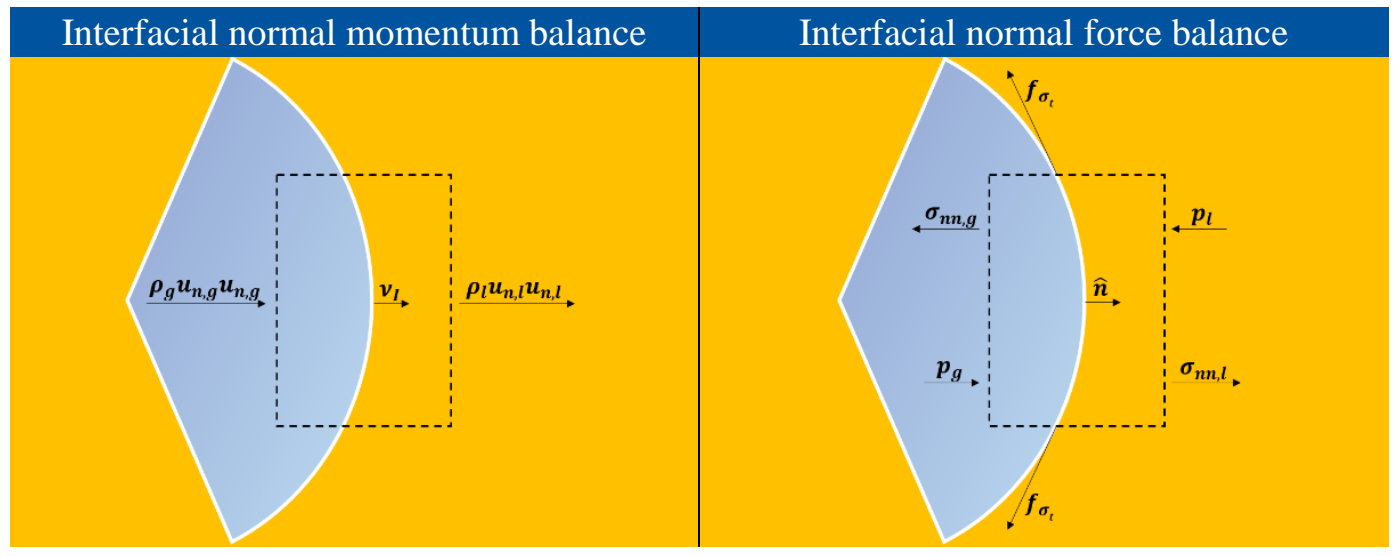

Fig. 5 Interfacial normal momentum and force balance

In practice, the jump in normal deviatoric stress is neglected [5] resulting in:

$$
[p]=\kappa_{c} \sigma_{t}-\rho_{l} r_{b}\left[u_{n}\right]
$$

Interfacial tangential momentum balance. The tangential momentum balance and the tangential force balance at an interface is depicted in Fig. 6.

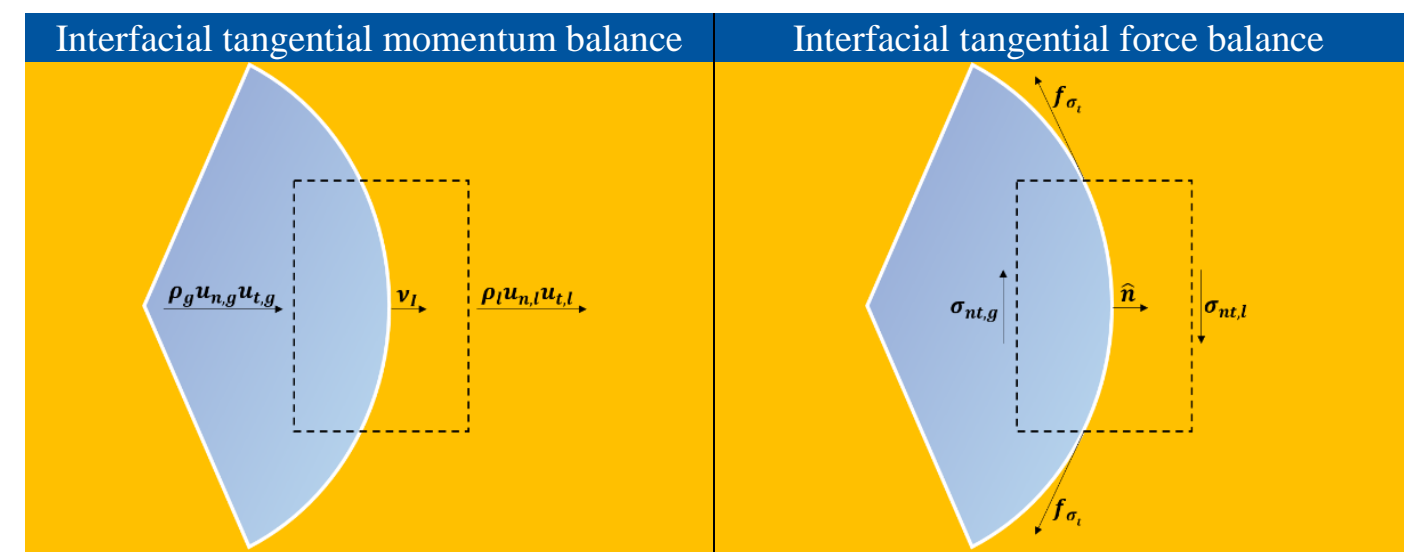

Fig. 6 Interfacial tangential momentum and force balance

Using Reynolds transport theorem and Newton's second law results in equation (19), in which the surface tension gradient in tangential direction is defined with equation (20).

$$
\begin{gathered}
-\rho_{g} u_{t, g}\left(u_{n, g}-u_{n, I}\right)+\rho_{l} u_{t, l}\left(u_{n, l}-u_{n, I}\right)=-\sigma_{n t, g}+\sigma_{n t, l}+\left\|\nabla_{s} \sigma_{t}\right\| \\
\nabla_{s} \sigma_{t}:=\nabla \sigma_{t}-\widehat{\boldsymbol{n}} \cdot\left(\widehat{\boldsymbol{n}} \cdot \nabla \sigma_{t}\right)
\end{gathered}
$$

By using the definition of the regression rate and defining the jump in tangential velocity, an expression for the jump in the deviatoric shear stress is derived:

$$
\left[\sigma_{n t}\right]=\rho_{l} r_{b}\left[u_{t}\right]+\left\|\nabla_{s} \sigma_{t}\right\|
$$

For large particles and droplets, the no-slip condition can be applied and equation (21) simplifies to:

$$
\left[\sigma_{n t}\right]=\left\|\nabla_{s} \sigma_{t}\right\|
$$


Interfacial species mass balance. Similarly, to the interfacial mass balance, an interfacial species mass balance can be made and is depicted in Fig. 7.

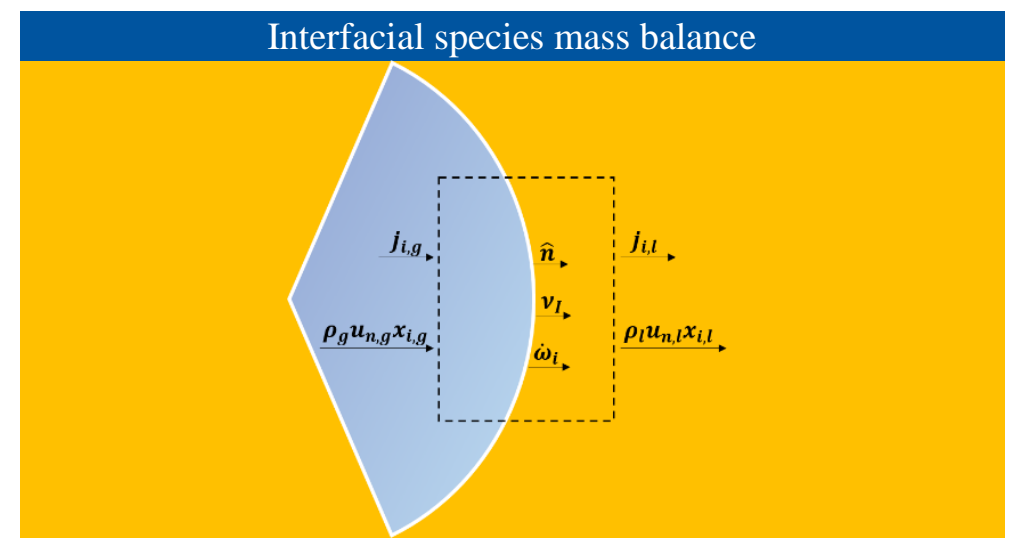

Fig. 7 Interfacial species mass balance

The resulting equation is given by:

$$
-x_{i, g} \rho_{g}\left(u_{n, g}-u_{n, I}\right)-j_{i, g}+x_{i, l} \rho_{l}\left(u_{n, l}-u_{n, I}\right)+j_{i, l}=\dot{\omega}_{i}
$$

From equation (23) follows, that at an interface species mass is not conservative in the presence of chemical reactions. If chemical reactions are neglected and the definition of the regression rate and the jump in normal velocity is used, the expression for interfacial species mass balance can be rewritten to:

$$
-x_{i, g} \rho_{g}\left(\left[u_{n}\right]+r_{b}\right)-j_{i, g}+x_{i, l} \rho_{l} r_{b}+j_{i, l}=0
$$

Interfacial energy balance. In a similar manner, an energy balance is made at the interface shown in Fig. 8 .

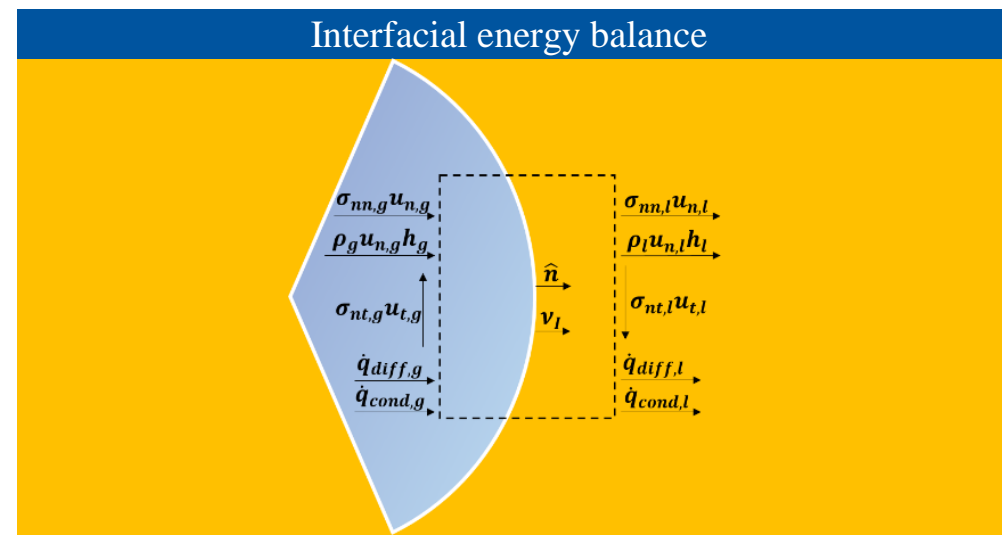

Fig. 8 Interfacial energy balance

The resulting equation for the interfacial energy balance is given by:

$$
\begin{gathered}
-\rho_{g} h_{g}\left(u_{n, g}-u_{n, I}\right)-\sigma_{n n, g} u_{n, g}-\sigma_{n t, g} u_{t, g}-\dot{q}_{c o n d, g}-\dot{q}_{d i f f, g}+\rho_{l} h_{l}\left(u_{n, l}-u_{n, I}\right)+\sigma_{n n, l} u_{n, l} \\
+\sigma_{n t, l} u_{t, l}+\dot{q}_{c o n d, l}+\dot{q}_{d i f f, l}=0
\end{gathered}
$$

The jump in heat flux due to conduction at an interface is given by:

$$
\left[\dot{q}_{c o n d}\right]=\rho_{l} r_{b}\left(h_{l}-h_{g}\right)-\left[\sigma_{n n} u_{n}\right]-\left[\sigma_{n t} u_{t}\right]-\left[\dot{q}_{d i f f}\right]
$$




\subsection{Assumptions and Simplifications}

To model gas-cavitation, the governing equations can be simplified to neglect phase change at the interface. This simplification is justified as the emphasis is placed on the bubble growth due to diffusion. With this assumption, according to Faghri et al. [7], the relative velocities are zero and equation (12) reduces to:

$$
\rho_{g}\left(u_{n, g}-u_{n, I}\right)=\rho_{l}\left(u_{n, l}-u_{n, I}\right)=0
$$

Furthermore, the jump in normal velocity vanishes:

$$
\left[u_{n}\right]=0
$$

The jump in pressure is due only to surface tension:

$$
[p]=\kappa_{c} \sigma_{t}
$$

Equation (24) for the species mass balance reduces to:

$$
j_{i, g}=j_{i, l}
$$

According to Bird et al. [8], concentration gradients can produce heat transfer, known as the diffusion-thermo effect, but its contribution to the total energy flux is usually small. Therefore, by neglecting the heat transfer due to the diffusion-thermo effect, equation (26) for the energy balance simplifies to:

$$
\left[\dot{q}_{\text {cond }}\right]=0
$$

\section{NUMERICAL APPROACH}

The presence of shock waves requires the use of special numerical methods. According to Toro [4], the mathematical formulation as well as the numerical scheme pose challenges to the convergence of the solution. One way of solving the governing equations is by the use of conservative upwind methods, where the integral formulation is used. Therefore, the finite volume method (FVM), also known as the control volume approach, is adopted in this work.

An outline of the numerical method is presented in the following chapters and a flowchart of the computational procedure is given at the end of this paper in Fig. 13.

\subsection{Numerical scheme}

To solve the Navier-Stokes equations, the fractional-step method, also known as dimensional and source term splitting, is used. This allows solving the hyperbolic Euler equations using a conventional Riemann-Solver and the parabolic terms using any numerical integration scheme for ordinary differential equations. A first-order accurate splitting scheme is given by equation (32).

$$
\boldsymbol{U}^{n+1}=\mathcal{P}_{x y}^{\Delta t} \mathcal{H}_{y}^{\frac{\Delta t}{2}} \mathcal{H}_{x}^{\frac{\Delta t}{2}}\left(\boldsymbol{U}^{n}\right)
$$

The splitting-scheme for the hyperbolic and parabolic operators is given by equations (33) to (35). 


$$
\begin{aligned}
& \mathcal{H}_{x}^{\frac{\Delta t}{2}}\left(\boldsymbol{U}^{n}\right):\left\{\begin{array}{cl}
P D E s: & \boldsymbol{U}_{t}+\boldsymbol{F}(\boldsymbol{U})_{x}=0 \\
I C s: & \boldsymbol{U}^{n}
\end{array} \Rightarrow \overline{\boldsymbol{U}}^{n+\frac{1}{2}}\right. \\
& \mathcal{H}_{y}^{\frac{\Delta t}{2}}\left(\boldsymbol{U}^{n}\right):\left\{\begin{array}{cl}
\text { PDES }: & \boldsymbol{U}_{t}+\boldsymbol{G}(\boldsymbol{U})_{x}=0 \\
\text { ICs }: & \overline{\boldsymbol{U}}^{n+\frac{1}{2}}
\end{array} \Rightarrow \overline{\boldsymbol{U}}^{n+1}\right. \\
& \mathcal{P}_{x y}^{\Delta t}\left(\boldsymbol{U}^{n}\right):\left\{\begin{array}{cl}
P D E s: & \frac{d}{d t}(\boldsymbol{U})=\frac{\partial \boldsymbol{F}_{\text {par }}}{\partial x}+\frac{1}{y^{\zeta}} \frac{\partial y^{\zeta} \boldsymbol{G}_{\text {par }}}{\partial y}-\zeta \frac{\boldsymbol{S}_{\text {geom }}}{y} \Rightarrow \boldsymbol{U}^{n+1} \\
I C s: & \overline{\boldsymbol{U}}^{n+1}
\end{array}\right.
\end{aligned}
$$

\subsection{Conventional Riemann-problem}

The structure of the solution of a conventional two-dimensional Riemann-problem is depicted in Fig. 9. For the Euler equations, 4 distinct regions are separated by 4 waves given by the eigenstructure of the $x$-split system of partial differential equations:

$$
\lambda_{1}=u-a \quad \lambda_{2}=u \quad \lambda_{3}=u \quad \lambda_{4}=u+a
$$

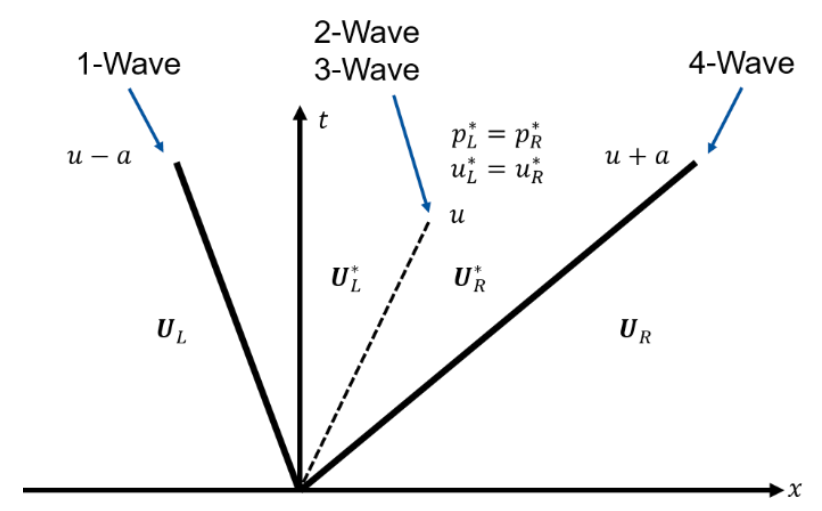

Fig. 9 Solution of two-dimensional conventional Riemann-problem

Across the 2-wave and the 3-wave both pressure and normal velocity remain constant but there is a discontinuous jump in density across the 2-wave and a discontinuous jump in tangential velocity across the 3-wave [4]. The 1-wave and 4-wave can be either a shock or a rarefication wave across which the tangential velocity remains constant [4]. For better understanding, the 2- and 3-waves will be collectively referred to as a contact discontinuity.

Due to the use of the fractional step method, the existing Riemann-problem is solved separately along each coordinate axis [9]. Toro [4] provides a good overview of exact and approximate Riemann-solvers. In this work, the approximate HLLC-solver is used for both gaseous and liquid phase.

The HLLC-solver is a robust approximate Riemann-solver which is applicable both to multidimensional and multicomponent flows [4]. Compared to, e.g. original solver of Roe or Roe-Pike method, the HLLC-solver does not require an entropy fix to handle unphysical rarefication shocks [4].

For a detailed discussion on Riemann-problems and Riemann-solvers, the author refers to Toro [4] and Leveque [9]. 


\subsection{Interfacial Riemann-problem}

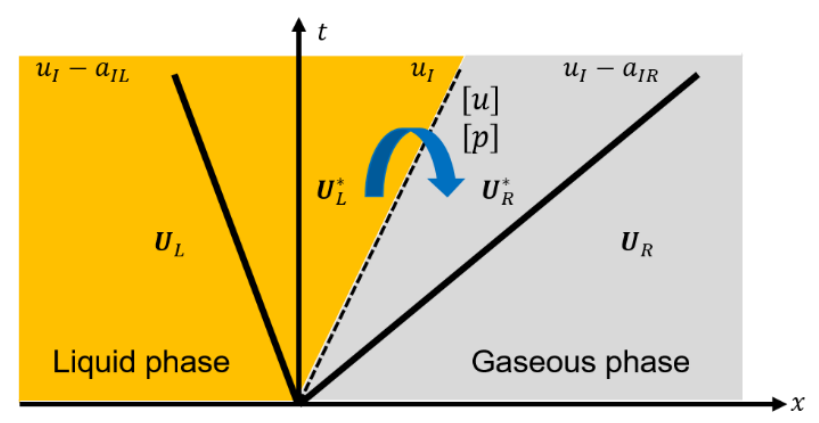

Fig. 10 Interfacial Riemann-problem

The interfacial Riemann-problem is shown in Fig. 10. Although the wave constellation is the same as for the conventional Riemann-problem, ordinary Riemann-solvers cannot be used directly. This is because, according to Houim et al. [5], normal velocity and pressure are no longer constant across the contact discontinuity when phase change and surface tension are considered, assumptions on which ordinary Riemann-solvers rely upon [4]. Hence, the jump conditions of chapter 2.2 must be applied:

$$
\begin{gathered}
u_{I}=u_{L}^{*}-r_{b} \\
u_{R}^{*}=u_{L}^{*}+[u] \\
p_{R}^{*}=p_{L}^{*}+[p]
\end{gathered}
$$

To solve the interfacial Riemann-problem, the exact Riemann-solver is used. Following the procedure of Toro [4] and Houim [10], velocity jump functions are defined to solve an implicit algebraic equation numerically for the pressure in the star-state:

$$
\begin{gathered}
f_{L}\left(p_{L}^{*}, u_{L}, p_{L}, \rho_{L}\right)+g_{R}\left(p_{R}^{*}, u_{R}, p_{R}, \rho_{R}\right)+u_{R}-u_{L}-[u]=0 \\
f_{L}= \begin{cases}\sqrt{\frac{p_{L}-p_{L}^{*}}{\rho_{L}^{*}-\rho_{L}}} & p_{L}<p_{L}^{*} \\
\int_{p_{L}}^{p_{L}^{*}} \frac{d p}{\rho a} & p_{L} \geq p_{L}^{*}\end{cases} \\
g_{R}=\left\{\begin{array}{cc}
\left(p_{R}^{*}-p_{R}\right) \sqrt{\frac{2}{(\gamma+1) p_{R}^{*}+(\gamma-1) p_{R}}} & p_{R}<p_{R}^{*} \\
\frac{2 a_{R}}{\gamma-1}\left(\frac{p_{R}^{*}}{p_{R}}\right)^{\frac{\gamma-1}{2 \gamma}}-\frac{2 a_{R}}{\gamma-1} & p_{R} \geq p_{R}^{*}
\end{array}\right.
\end{gathered}
$$




\subsection{Level-set method}

To track the position of the interface, a level-set method is used. Osher et al. [11] and Sethian [12] define the implicit level-set function $\varphi$ using the advection equation:

$$
\frac{\partial \varphi}{\partial t}+\boldsymbol{v} \cdot \nabla \varphi=0
$$

Equation (43) defines the motion of an interface where:

$$
\varphi(\boldsymbol{x})=0
$$

By using a signed distance function with the properties (45) and (46), steep and flat gradients are avoided as much as possible, which gives better numerical results [11]. The level-set function for a circle is depicted in Fig. 11.

$$
\begin{gathered}
|\nabla \varphi|=1 \\
\varphi(\boldsymbol{x})=\left\{\begin{array}{cc}
d(\boldsymbol{x}) & \forall x \in \Omega^{+} \\
0 & \forall x \in \partial \Omega \\
-d(\boldsymbol{x}) & \forall \boldsymbol{x} \in \Omega^{-}
\end{array}\right.
\end{gathered}
$$

The use of a level-set method has certain advantages over other methods. In contrast to front tracking methods, where a finite set of points are used to evolve the interface over time, level-set methods use the level-set function to represent and to evolve the interface using equation (43) [11]. A fundamental drawback of front tracking methods are the large distortions of surface elements in the most basic velocity fields [11]. Special procedures have to be implemented to smoothen and regularize these, whereas level-set methods just need to be reinitialized after a few time-steps to a new signed distance function [12].

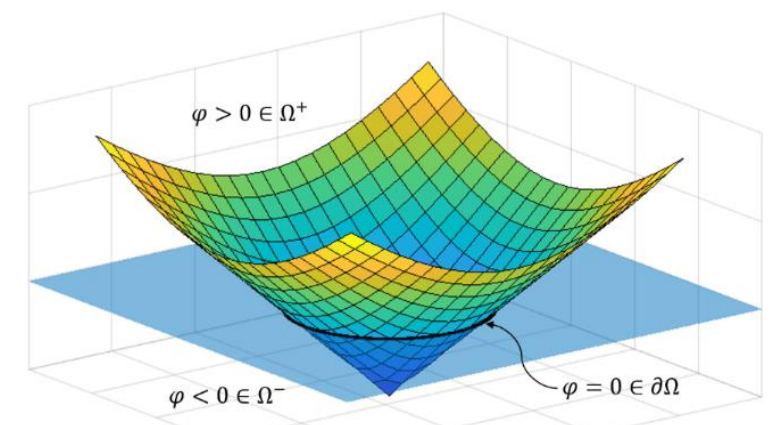

Fig. 11 Level-set function

\subsection{Ghost fluid method}

Fedkiw et al. [13] first proposed the ghost fluid method to solve multiphase flows. Parallel to the real grid of finite volume cells, they proposed to define an additional ghost grid that contains the ghost mass, momentum and energy for the other fluid that does not actually exist there.

First, the interfacial Riemann-problem is solved at the closest intercell boundary to the real interface. Then, a ghost grid is populated with the star-state values as shown in Fig. 12. This allows the liquid phase solver to compute in the gas domain as it were a liquid. Conversely, the gas phase solver computes in the liquid domain. 
Using this methodology, the correct jump conditions are captured and a velocity field around the interface can be defined [13]. Using this velocity field, the interface can then be advected within the level-set method framework. Depending on the new position of the interface, the value of the level-set function defines which cells belong to the liquid phase and which to the gas phase.

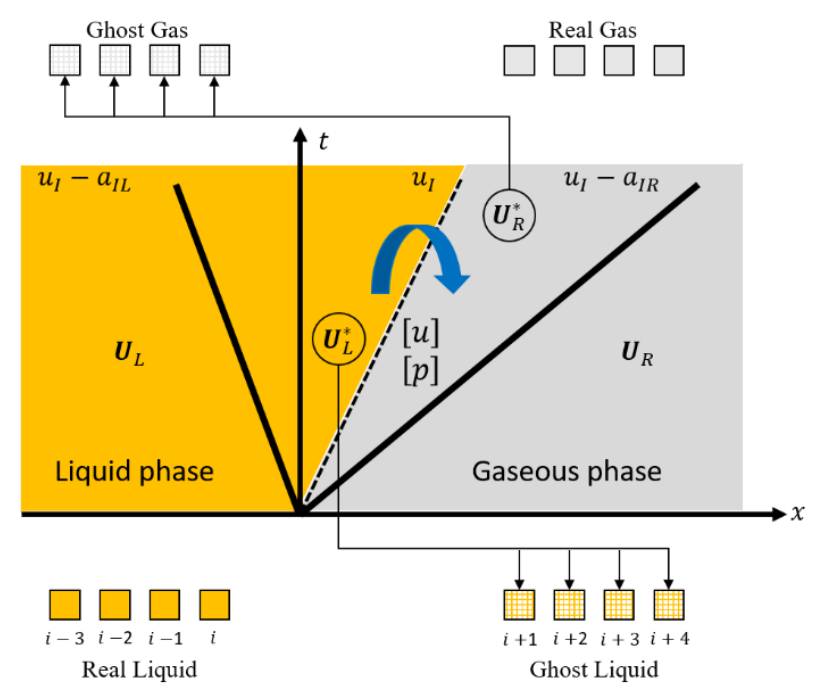

Fig. 12 Interfacial Riemann-problem

$+$

\section{PROPOSED VALIDATION STRATEGY}

Several test cases are proposed to validate the simulation model. For hyperbolic solvers, the original Sod's test and the modified Sod's test provide assessment of the entropy condition; the so-called 123 problem allows testing for low-density flows [4]. In addition, the Noh problem and the Sedov problem are proposed to validate the numerical capacity and convergence of the hyperbolic solver [14].

Following the validation of the hyperbolic solver for single phase flows, a gas-water shock tube problem and a test, where a shock wave impacts a gas-water interface are proposed to validate the multiphase capacity of the computational model [15].

The parabolic solver should be validated in two steps. First, the viscosity model should be validated by comparing the computed numerical results with known analytical solutions of different fluid flows, e.g. the flow in a pipe. To that end, heat and mass transfer is disabled.

Secondly, to validate the thermal and mass diffusion model, it is proposed that the hyperbolic solver, which is used to compute fluid flow, be disabled. As a result, the parabolic solver can be validated by comparing the numerical results with known analytical solutions of classic heat and mass transfer problems, e.g. heat transfer in a plane wall, mass diffusion in a gas column.

\section{CONCLUSIONS}

In hydraulic systems, the phenomenon of cavitation can lead cavitation erosion damage as well as increase the risk of the micro-diesel effect. Although different cavitation models exist, there is no model of which the author is aware of at the time of this publication that accounts for gas-cavitation, i.e., the diffusion-driven growth of gas bubbles. 
To model gas-cavitation, a computational model for a single gas bubble immersed in liquid based on the interfacial Riemann-problem is proposed. To that end, the Navier-Stokes equations of each phase are coupled at the interface.

The numerical coupling of both phases is achieved by utilizing the ghost fluid method within a level-set method framework. This allows the gas solver and the liquid solver to resolve the flow field on a ghost grid and calculate the sought after values for the conserved variables. To move the interface after one time step, the level-set method is used.

Using already resolved test cases from fluid dynamics, heat and mass transfer and water-gas shock tube problems, the multiphase capacity of the proposed model can be validated.

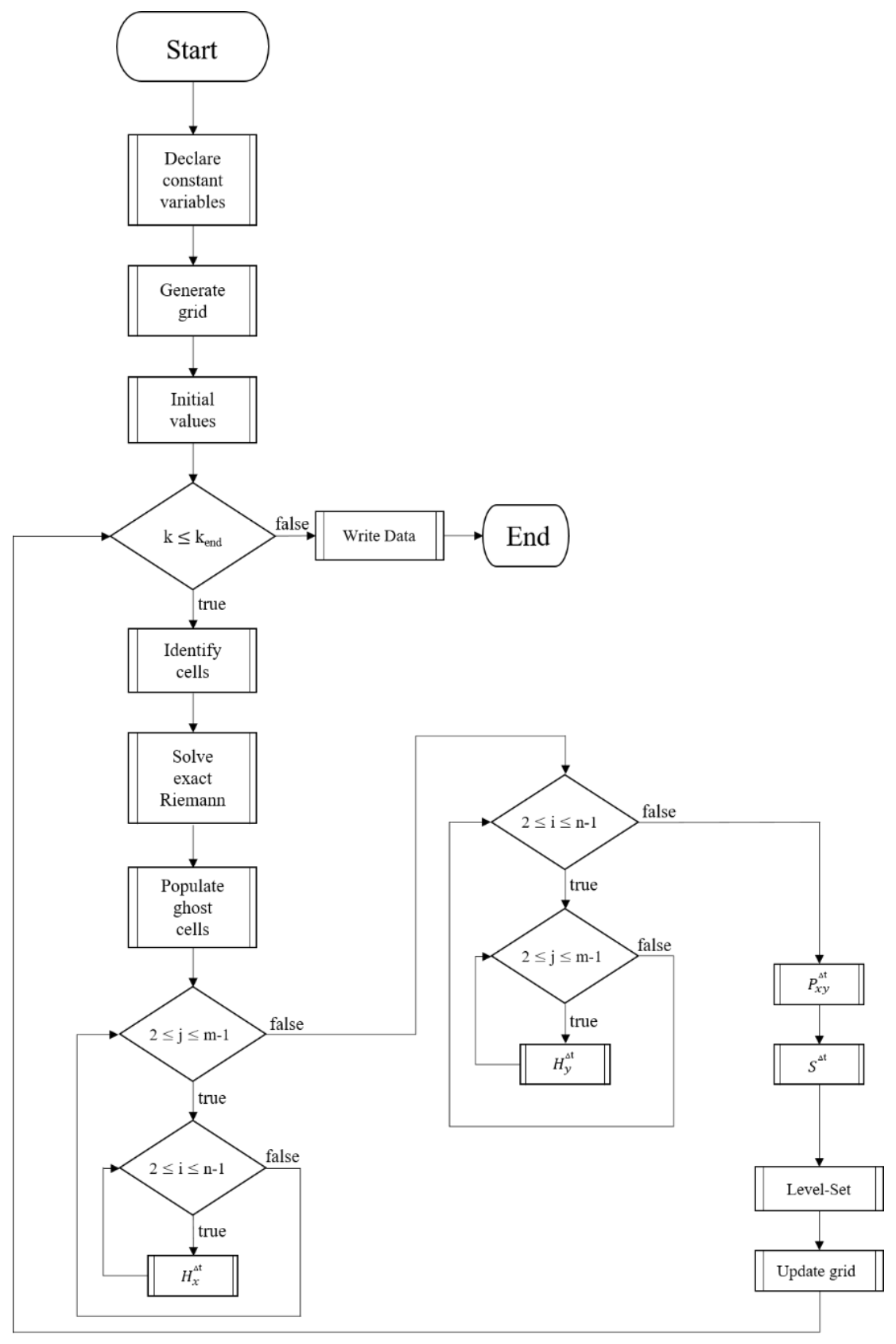

Fig. 13 Computational procedure 


\section{ACKNOWLEDGMENT}

This work was funded by the German Research Foundation (DFG) in the scope of the Project „Lösungs- und Entlösungsverhalten von Luft in Hydraulikölen" (MU 1225/41-1). The authors would like to thank the DFG for its support.

\section{NOMENCLATURE}

$\begin{array}{llllll}A & \text { parameter / surface area } & \left(\mathrm{bar} / \mathrm{m}^{2}\right) & \boldsymbol{v} & \text { velocity vector } & (\mathrm{m} / \mathrm{s}) \\ a & \text { speed of sound } & (\mathrm{m} / \mathrm{s}) & x & \text { x-coordinate } & (\mathrm{m}) \\ B & \text { Tait equation of state parameter } & (\mathrm{bar}) & \boldsymbol{x} & \text { coordinate vector } & (\mathrm{m}) \\ B_{\text {sys }} & \text { extensive property } & & \bar{x} & \text { molar fraction } & (-) \\ b & \text { intensive property } & & y & \text { y-coordinate } & (\mathrm{m}) \\ d(\boldsymbol{x}) & \text { signed distance function } & & & & \\ E & \text { total energy } & \left(\mathrm{J} / \mathrm{m}^{3}\right) & \gamma & \text { specific heat ratio / parameter } & (-) \\ H & \text { Henry's law constant } & (\mathrm{bar}) & \zeta & \text { coordinate selector variable } & (-) \\ h & \text { specific enthalpy } & (\mathrm{J} / \mathrm{mol}) & \kappa_{C} & \text { curvature } & (1 / \mathrm{m}) \\ j & \text { diffusion flux } & \left(\mathrm{kg} / \mathrm{m}^{2} \mathrm{~s}\right) & \lambda & \text { eigenvalue } & (\mathrm{m} / \mathrm{s}) \\ \dot{m}_{I}^{\prime \prime} & \text { mass flux } & \left(\mathrm{kg} / \mathrm{m}^{2} \mathrm{~s}\right) & v & \text { kinematic viscosity } & \left(\mathrm{m}^{2} / \mathrm{s}\right) \\ \widehat{\boldsymbol{n}} & \text { unit normal vector } & (-) & \rho & \text { density } & \left(\mathrm{kg} / \mathrm{m}^{3}\right) \\ p & \text { pressure } & (\mathrm{bar}) & \sigma & \text { deviatoric stress tensor } & (\mathrm{Pa}) \\ q & \text { heat flux } & \left(\mathrm{W} / \mathrm{m}^{2}\right) & \sigma_{t} & \text { surface tension } & (\mathrm{N} / \mathrm{m}) \\ R & \text { radius } & (\mathrm{m}) & \varphi & \text { level-set function } & (\mathrm{m}) \\ r_{b} & \text { regression rate } & (\mathrm{m} / \mathrm{s}) & \Psi & \text { general variable } & \\ t & \text { time } & (\mathrm{s}) & \dot{\omega} & \text { species production rate } & \left(\mathrm{kg} / \mathrm{m}^{3} \mathrm{~s}\right) \\ u & \text { velocity } & (\mathrm{m} / \mathrm{s}) & & & \\ V & \text { volume } & (\mathrm{m} 3) & & & \end{array}$

\section{REFERENCES}

[1] Lüdecke, C., Lüdecke, D., Thermodynamik: Physikalisch-chemische Grundlagen der thermischen Verfahrenstechnik, Berlin: Springer-Verlag, (2000)

[2] Brennen, C.E., Cavitation and bubble dynamics, New York: Oxford University Press, (1995)

[3] Schmitz, K., Murrenhoff, H., Grundlagen der Fluidtechnik: Hydraulik, Aachen: Shaker Verlag, (2018)

[4] Toro, E.F., Riemann solvers and numerical methods for fluid dynamics: a practical introduction, Dordrecht: Springer-Verlag, (2009)

[5] Houim, R.W., Kenneth, K.K., "A ghost fluid method for compressible reacting flows with phase change," J. Computational Physics, 235, pp. 865-900, (2013).

[6] Mamoru, I., Takashi, H., Thermo-Fluid Dynamics of Two-Phase Flow, New York: Springer-Verlag, (2006)

[7] Faghri, A., Zhang, Y., Transport phenomena in multiphase systems, Amsterdam: Elsevier, (2006)

[8] Bird, R.B., Stewart, W.E., Lightfoot, E.N., Transport phenomena, New York: John Wiley \& Sons, (2007)

[9] Leveque, R.J., Finite volume methods for hyperbolic problems, Cambridge: Cambridge University Press, (2007)

[10] Houim, R.W., Modeling the influence of shock waves on the combustion of aluminum droplets, Ph.D. Thesis, Pennsylvania State University, (2011).

[11] Osher, S., Fedkiw, R.P., Level Set Methods and Dynamics Implicit Surfaces, New York: Springer-Verlag, (2003)

[12] Sethian, J.A., "Evolution, Implementation, and Application of Level Set and Fast Marching Methods for Advancing Fronts," $J$. Computational Physics, 169(2), pp. 503-555, (2001)

[13] Fedkiw, R.P., Aslam, T., Merriman, B., Osher, S., "A Non-oscillatory Eulerian Approach to Interfaces in Multimaterial Flows (the Ghost Fluid Method)," J. Computational Physics, 152(2), pp. 457-492, (1999)

[14] Doebling, S.W., Standardized Definitions for Code Verification Test Problems. Retrieved 10.04.2019 from https://permalink.lanl.gov/object/tr?what=info:lanl-repo/lareport/LA-UR-17-28269, (09.14.2017)

[15] Liu, T.G., Khoo, B.C., Yeo, K.S., "Ghost fluid method for strong shock impacting on material interface," J. Computational Physics, 190(2), pp. 651-681, (2003) 